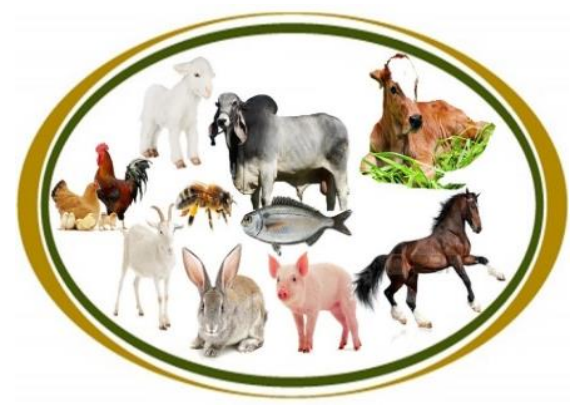

Recepción: 16 de abril de 2020

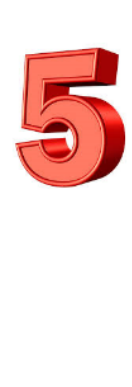

Oracultad de Ciencias

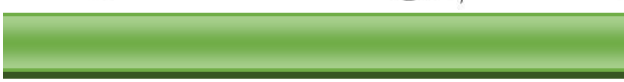

ISSN L 2644-3856

Revista Investigaciones Agropecuarias

Volumen 2, $N^{\circ}$ 2. pp. 69-85

Junio 2020 - Noviembre 2020

Panamá

Aceptación: 8 de mayo de 2020

\title{
EFECTO DE LA DOMA RACIONAL SOBRE EL TEMPERAMENTO, RELACIÓN HUMANO-ANIMAL Y DESEMPEÑO PRODUCTIVO EN NOVILLAS CRUZADAS
}

\author{
Joseph Grajales Cedeño' ${ }^{\prime}$, Hernán Atencio ${ }^{1}$, Reynaldo Vargas $^{1,3}$, Alex Ríos Moreno ${ }^{2,3}$, Efraín Quintero ${ }^{4}$, Héctor \\ Cedeño ${ }^{\prime}$, Javier Bethancourt ${ }^{1,5}$
}

\footnotetext{
1* Universidad de Panamá, Facultad de Ciencias Agropecuarias, Departamento de Zootecnia. Panamá

${ }^{2}$ Universidad de Panamá, Facultad de Ciencias Agropecuarias, Departamento de Protección Vegetal. Panamá

${ }^{3}$ Sistema Nacional de Investigación (SNI) de Panamá- Senacyt. Panamá

${ }^{4}$ Servicio de Reproducción Animal SRA, S.C. Panamá.

${ }^{5}$ Universidad Federal de Lavras, Doctorando en Zootecnia, Brasil
}

*joseph.grajales@up.ac.pa, hernan-8804@hotmail.com, rvargas661@gmail.com, reneejjr@gmail.com, hector22d@gmail.com, javierbethancourt_@hotmail.com

alex.morenom@up.ac.pa,

\section{RESUMEN}

Evaluamos el impacto de la doma racional sobre el temperamento, relación humano animal y desempeño productivo en novillas cruzadas. Este estudio se realizó en el Centro de Investigaciones en Biotecnología Agropecuaria, de la Facultad de Ciencias Agropecuarias, de la Universidad de Panamá. Se utilizaron 16 animales divididos al azar en dos grupos. Grupo con doma racional: $(\mathrm{n}=8)$ con un peso de $260.63 \pm 17.11 \mathrm{~kg}$ y edad de $11.0 \pm 1.0$ meses. Grupo con doma tradicional: $(n=8)$ con un peso de $264.88 \pm 24.07$ y edad de $11.63 \pm 0.75$ meses. Los datos se analizaron con el programa estadístico STATISTICA V10 y GraphPad Prism V8.0.2. Se empleo prueba no paramétrica de Kruskal-Wallis, Mann-Whitney U y la prueba no paramétrica Q de Cochran para score binarios. De manera general hubo diferencias en cuanto al temperamento en función del tiempo en ambos grupos $(\mathrm{p}<0.01)$. Al día 0 y 16 del estudio los animales tenían un score de agresividad de 70 y $20 \%$ respectivamente $(p=0.04)$. Sin embargo, en el grupo de doma tradicional el temperamento no cambio $(\mathrm{p}=0.15)$. De manera general la relación humano-animal también mostró diferencias significativas en ambos grupos ( $<$ 0.05). La ganancia diaria de peso dieciséis días posteriores al inicio del estudio para el grupo de doma racional y tradicional fue de 0.6 y $0.06 \mathrm{~kg} / \mathrm{d}$ respectivamente $(\mathrm{p}>0.05)$. No hubo diferencias ( $p>0.05$ ) en el peso acumulado al final del periodo de estudio. En conclusión, la doma racional tuvo un efecto positivo sobre el temperamento, la relación humano-animal y el rendimiento productivo de las novillas.

PALABRAS CLAVES: Temperamento, Bienestar animal, Doma racional, Comportamiento, Relación humano-animal 


\section{EFFECT OF TWO DOMA METHODS ON TEMPERAMENT, HUMAN-ANIMAL RELATIONSHIP AND PRODUCTIVE PERFORMANCE IN CROSSED HEIFERS}

\section{ABSTRACT}

The objective of this study was to evaluate the impact of rational dressage on temperament, human-animal relationship and productive performance in heifers. This study was carried out at the Center for Research in Agricultural Biotechnology, of the Faculty of Agricultural Sciences, of the University of Panama. Sixteen animals divided randomly into two groups were used. Group with rational dressage: $(n=8)$ with a weight of $260.63 \pm 17.11 \mathrm{~kg}$ and age of $11.0 \pm 1.0$ months. Traditional dressage group: $(n=8)$ with a weight of $264.88 \pm 24.07$ and age of $11.63 \pm 0.75$ months. The data were analyzed with the STATISTICA V10 statistical program and GraphPad Prism V8.0.2. Kruskal-Wallis non-parametric test, Mann-Whitney U and Cochran Q non-parametric test were used for binary scores. In general, there were differences in terms of temperament as a function of time in both groups $(\mathrm{p}<0.01)$. At day 0 and 16 of the study, the animals had an aggressiveness score of 70 and $20 \%$ respectively $(p=0.04)$. However, in the traditional dressage group the temperament did not change $(p=0.15)$. In general, the human-animal relationship also showed significant differences in both groups $(\mathrm{p}<0.05)$. The daily weight gain sixteen days after the start of the study for the rational and traditional dressage group was 0.6 and $0.06 \mathrm{~kg} / \mathrm{d}$ respectively $(\mathrm{p}>0.05)$. There were no differences $(p>0.05)$ in the accumulated weight at the end of the study period. In conclusion, rational taming had a positive effect on the temperament, the human-animal relationship and the productive performance of the heifers.

KEYWORDS: Temperament, Animal welfare, Rational dressage, Behavior, Human-animal relationship 


\section{INTRODUCCIÓN}

Existe una variación significativa en cómo los animales se comportan y responden a su entorno. Por lo cual recientemente ha habido un creciente interés en la investigar el comportamiento ya que, dentro de la producción pecuaria puede impactar directamente en el bienestar y mermar el potencial de producción. Los programas de producción pecuaria modernos deben ofrecer productos de calidad, con un componente de sustentabilidad y la promoción del bienestar humanoanimal en armonía con el medio ambiente (García-Pinillos et al., 2015). En ese sentido el estudio del comportamiento y el bienestar animal proporciona una nueva perspectiva para el modelo convencional de producción.

El termino temperamento se ha definido como como el temor y reactividad a los humanos o ambientes nuevos o amenazantes (Burrow, 1997; Grandin, 1993a), la manera en que un individuo reacciona a una situación nueva o desafiante y que son que son consistentes a lo largo del tiempo y/o en contextos (Hedlund y Løvlie, 2015; Koolhaas et al., 1999; Reale et al., 2000). La demostración más visible del temperamento de un animal es su reactividad, influenciada por miedo que siente el animal frente a la percepción del peligro (Grignard et al., 2001). Los estudios reportan que el temperamento tiene repercusiones en la producción de leche (Breuer et al., 2000; Cziszter et al., 2016; Sutherland y Dowling, 2014), calidad de la carne, tasas de crecimiento en ganado de carne y doble propósito (Cafe et al., 2011; Reinhardt et al., 2009; Turner et al., 2011), así como en el desempeño reproductivo (Cooke et al., 2017; Haile-Mariam et al., 2004; Sewalem et al., 2011) y longevidad (Haskell et al., 2014; Sewalem et al., 2010) en bovinos.

La raza Bos taurus indicus y sus cruces presentan mayor reactividad, a pesar de existir variaciones entre individuos. Ante esta situación es posible mejorar la reactividad o temperamento del ganado con la implementación de buenas prácticas, como lo es la doma y el manejo racional, en ese sentido la literatura reporta que la relación humano-animal es uno de los factores que impactan directamente en el bienestar del ganado (Hemsworth, 2007), afectando características productivas y de comportamiento (Coleman y Hemsworth, 2014). En Panamá aún no se han realizado estudios científicos con el abordaje antes descrito. Por lo tanto, el objetivo de este estudio fue evaluar el impacto de la doma racional sobre el temperamento, relación humano animal y desempeño productivo en novillas de carne bajo condiciones pastoriles.

\section{MATERIALES Y MÉTODOS}

\section{Animales, condiciones de alojamiento y manejo}

Este estudio se realizó en el Centro de Investigaciones en Biotecnología Agropecuaria, de la Facultad de Ciencias Agropecuarias, de la Universidad de Panamá, ubicado en el corregimiento

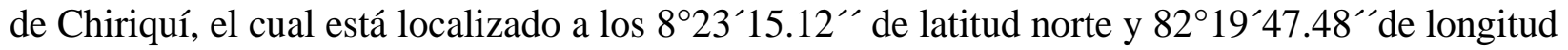


oeste, con una elevación de 26 msnm.

Se utilizaron animales cruzados (5/8 Simmental x 3/8 Brahman), alojados en sistemas pastoriles, su alimentación se basaba principalmente en Brachiaria humidicola y Brachiaria brizantha, suplementación mineral y agua ad libitum.

La rutina de manejo para cada día de ensayo consistía en transportar los animales por dos vaqueros a caballo desde las parcelas de pastoreo hacia los corrales donde recibían cada tratamiento según el método de doma empleado.

\section{Métodos de doma a emplear}

\section{La doma racional}

Los manejadores fueron entrenados previamente en la doma racional y en buenas prácticas de manejo de ganado vacuno. El entrenamiento incluyó dos componentes principales (similar a la metodología descrita por Hemsworth et al., 2002): 1) una sesión teórica sobre el comportamiento y 2) la aplicación del concepto de doma y manejo racional que tendrá como objetivo modificar las creencias y conductas, acompañado de una sesión de entrenamiento práctico sobre las mejores técnicas para el manejo del ganado. En este caso los animales fueron desensibilizados empleando sogas y unas varas unidas a un saco como extensión de nuestro cuerpo con la finalidad de reducir la zona de fuga y generar confianza en el animal a través de acciones positivas. El principio de este método se basó en observación del comportamiento de los animales y la no violencia a través de lecciones progresivas y repetitivas. Una vez desensibilizados y reducida la zona de fuga se procedió a colocar los bozales con el fin de que aprendieran a ser guiados por el manejador; posturas adecuadas (quedarse parados de manera correcta) y recibir manejo diario (baños y cepillados). Cada vez que el animal respondía de buena manera a los comandos se reforzaba positivamente con masajes.

\section{Doma tradicional}

En el caso de este grupo el manejo de los animales se caracterizó por el empleo de las prácticas comunes en fincas ganaderas como son el uso de acciones negativas al momento del manejo que generan estrés, miedo, dolor y muchas veces accidentes; siendo estos: contactos físicos agresivos, gritos, uso de corriente eléctrica para movilizar los animales, tirones, extrema agitación (Santos et al., 2015).

\section{Variables dependientes para evaluar}

Los animales se mantuvieron en los corrales durante aproximadamente 20 minutos para aclimatarse a las nuevas condiciones. Luego, los manejadores (ahora a pie) conducían los animales (6-8) al corral o manga de apartación para que caminaran a lo largo del conducto de trabajo (mangas) y entren en el conducto de compresión (brete), donde se tomaron las medias biométricas 
y de temperamento.

El temperamento fue evaluado por una sola persona utilizando el score de salida. El mismo se realizó al momento que el animal salió del brete de contención, en donde se denotó con $0=a$ un animal con temperamento adecuado (salida lenta y caminando) y $1=$ para animales agresivos (salida rápida, corre o trota) (Cooke et al., 2012; Grandin, 1993 a, b). Además, se utilizó el score de movimiento en brete de contención en dirección hacia un espacio abierto del corral adaptado la metodología empelada por (Cooke et al., 2011), en base a una escala de 5 puntos donde: (1) tranquilo, (2) movimientos mínimos, (3) movimientos frecuentes con vocalización, (4) movimientos constantes, vocalización y sacudidas; (5) lucha violenta y continua.

Relación humano-Animal (Acciones negativas). Se evaluó adaptando la metodología de Ceballos et al. (2018), que incluyen: golpear la puerta contra el cuerpo del animal, golpear o pinchar a la novilla con un palo de madera y torcer la cola del animal para que el animal ingrese al brete de contención. Se denotó con $0=$ a la usencia de acciones negativas y $1=$ para la presencia de acciones negativas.

Desempeño productivo: fue evaluado mediante la ganancia diaria de peso $(\mathrm{kg} / \mathrm{d})$ y a través del peso $(\mathrm{kg})$ al final del estudio.

\section{Análisis estadístico}

Se utilizo un diseño completamente al azar empleando el siguiente modelo: $Y_{i}=\mu+D_{i}+e_{i}$; Donde $Y_{i}=$ variable dependiente; $\mu=$ media; $D_{i}=$ efecto de la doma (Racional vs tradicional); $e_{i}=$ error aleatorio.

Se utilizó el software STATISTICA versión 10 (StatSoftV10) y Graph Pad Prism V.8.0.2 (San Diego, CA, USA). Los datos fueron sometidos a evaluación utilizando la prueba de Shapiro-Wilk's para analizar si contaban con una distribución normal y con la prueba de Levene's para analizar la homogeneidad de varianzas. Todas las variables evaluadas no mostraron distribución normal. Interacciones entre el tipo de doma y el tiempo fueron analizadas utilizando la prueba no paramétrica de Kruskal-Wallis. Este análisis fue seguido de una prueba de Dunn's, para identificar posibles diferencias entre grupos. Los resultados fueron expresados como Media \pm EE (error estándar). Además, se empleó la prueba no paramétrica Mann-Whitney U para comparar dos grupos, la prueba de Chi-cuadrado y la prueba no paramétrica $\mathrm{Q}$ de Cochran para score binarios (1=presencia; 0=Ausencia) (Oyeka, 2010). Se utilizó un alfa de 0.05. La consistencia entre temperamento (Score de salida y score de brete) fueron analizadas utilizando el coeficiente de correlación de Spearman.

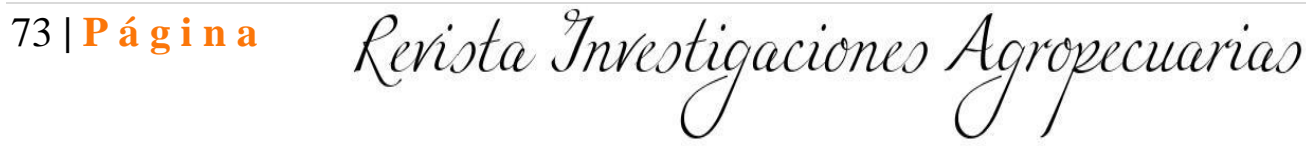




\section{Diseño experimental}

Con el fin de evaluar el temperamento, relación humano-animal y el desempeño utilizando dos métodos de doma se dividieron a los animales en dos grupos al azar de la siguiente manera:

Grupo con doma racional: conformado por 8 hembras con un peso promedio de $260.63 \pm 17.11$ $\mathrm{kg}$ y una edad promedio de $11.0 \pm 1.0$ meses.

Grupo con doma tradicional: animales que recibieron la doma convencional o tradicional, conformado por 8 hembras con un peso promedio de $264.88 \pm 24.07$ y una edad promedio de $11.63 \pm 0.75$ meses.

\section{RESULTADOS Y DISCUSIÓN}

\section{Efecto del método de doma sobre el temperamento (score de salida) e interacción humano- animal (interacciones negativas)}

\section{Doma racional}

De manera general hubo diferencias significativas en el temperamento (agresividad), medido a través del score de salida en los días de muestreo $(\mathrm{Q}=17.71 ; \mathrm{p}<0.001)$. Dieciséis días después de iniciado el ensayo los animales mostraban una reducción significativa de $70 \%$ a $20 \%$ en el score de temperamento $(\mathrm{Q}=4.0 ; \mathrm{p}=0.04)$. El temperamento se mantuvo bajo día $16(20 \%)$ y $32(10 \%)$ $(\mathrm{Q}=1.0 ; \mathrm{p}=0.31)$. Por su parte al día 30 y 62 fue de 10 y $0 \%$ respectivamente $(\mathrm{Q}=1.0 ; \mathrm{p}=0.31)$. Finalmente, el día 62 y 85 los animales no mostraron agresividad (Figura 1A).

De manera general la relación humano-animal medida a través de las acciones negativas mostró diferencias estadísticamente significativas $(\mathrm{Q}=9.81 ; \mathrm{p}=0.04)$. Las acciones negativas 16 días posterior de iniciado el estudio mostró una disminución significativa de $40 \%$ a un $10 \%(\mathrm{Q}=4.0$; $\mathrm{p}=0.04)$. Para el día 16 y 32 no hubo cambios $(10 \%$ para ambos $)(\mathrm{Q}=1.00 ; \mathrm{p}=0.31)$. Al día 30 y 62 fue de 10 y $0 \%$ respectivamente $(\mathrm{Q}=0.00 ; \mathrm{p}=1.0)$. Finalmente, el día 62 y 85 las acciones negativas fueron de 10 y $0 \%$ respectivamente $(\mathrm{Q}=1.0 ; \mathrm{p}=0.31)$. 
(A)

Temperamento $(\mathbf{p}<0.001)$

Relación humano-animal $(p=0.04)$
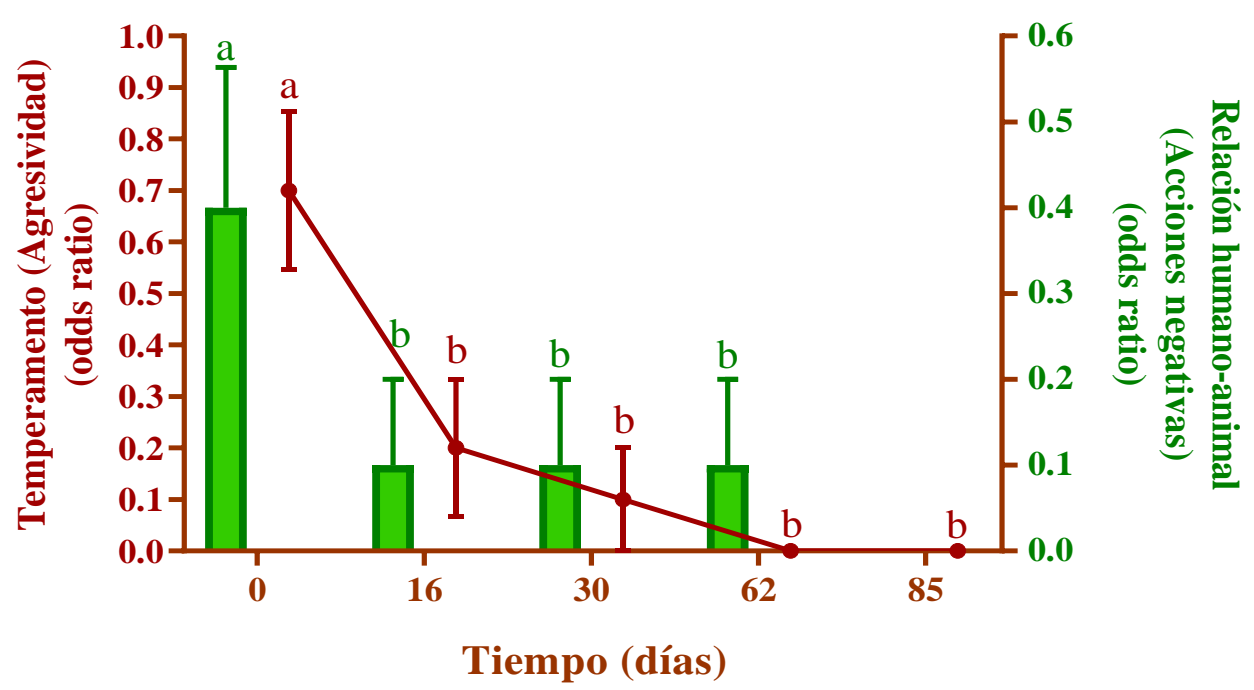

(B)

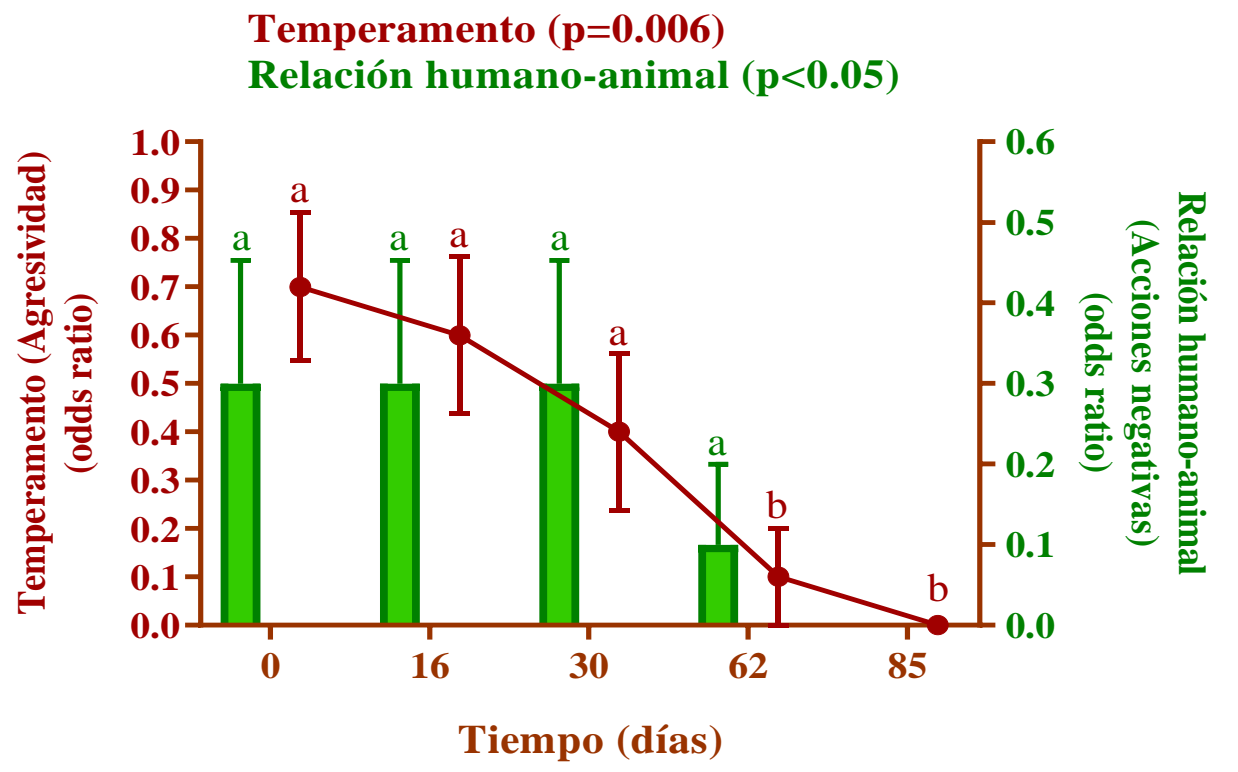

Figura 1. Media \pm error estándar del temperamento (score de salida) e interacción humano-animal (interacciones negativas) para da doma racional (A) y media \pm error estándar del temperamento (score de salida) e interacción humano-animal (interacciones negativas) para da doma tradicional (B). Letras diferentes ${ }^{(a, b)}$ indican diferencias estadísticamente significativas $(p<0.05)$. 


\section{Doma tradicional}

De forma general hubo diferencias significativas en temperamento (agresividad) medido a través del score de salida en los días de muestreo $(\mathrm{Q}=14.11 ; \mathrm{p}=0.006)$. Sin embargo, el temperamento 16 días posterior de iniciado el estudio no cambio $70 \%$ y $60 \%$ respectivamente $(\mathrm{Q}=2.0 ; \mathrm{p}=0.15)$. El temperamento se mantuvo similar, siendo de $60 \%$ al día 16 y $40 \%$ al día $32(\mathrm{Q}=0.00 ; \mathrm{p}=1.0)$. Por su parte al día 32 y 62 fue de $40 \%$ y $10 \%$ respectivamente $(Q=3.0 ; p=0.08)$. Finalmente, el día 62 y 85 la agresividad del animal fue de 10 y $0 \%$ respectivamente $(\mathrm{Q}=1.0 ; \mathrm{p}=0.31)$. De manera general la relación humano-animal mediada a través de las acciones negativas también mostró diferencias estadísticamente significativas $(\mathrm{Q}=4.85$; $\mathrm{p}<0.05)$. Sin embargo, no hubo cambios en las acciones negativas 16 días posterior de iniciado el estudio cambio de $30 \%$ en ambos casos $(\mathrm{Q}=0.33$; $\mathrm{p}=0.56)$. Para el día 16 y 32 tampoco hubo cambios $(30 \%$ para ambos $)(\mathrm{Q}=0.33 ; \mathrm{p}=0.56)$. $\mathrm{Al}$ día 30 y 62 fue de 30 y $10 \%$ respectivamente $(\mathrm{Q}=2.0 ; \mathrm{p}=0.15)$. Finalmente, el día 62 y 85 las acciones negativas fueron de 10 y $0 \%$ respectivamente $(Q=1.0 ; p=0.31$ ) (Figura 1B).

Un resultado interesante es que el temperamento en el grupo de doma racional cambio significativamente al día dieciséis, mientras que en el grupo de doma tradicional ese cambio se produjo sesenta y dos días después. Esta misma situación ocurrió para la relación humano-animal (acciones negativas),

Uno de los objetivos de los ganaderos es presentar de alto valor genético en las exposiciones ganaderas, sin embargo; para esto hay una preparación previa de los ejemplares; tal como amansarlos, que aprendan a caminar y colocarse correctamente en las pistas de juzgamientos o centros de exposición. Sin embargo, en muchos casos durante esta preparación los manejadores del ganado someten a los mismos a condiciones de estrés a través de golpes, gritos y maltrato, generando un pobre bienestar para ambos, lo cual repercute directamente en el comportamiento, bienestar y desempeño de los animales. Los resultados de este estudio indican que la doma racional tuvo un efecto positivo sobre el temperamento. Se observa que, al inicio del estudio los animales comenzaron con scores altos de agresividad de $70 \%$ y al cabo de dieciséis días el score bajo a un $20 \%$ y al mes un $10 \%$. Por su parte las acciones negativas también fueron desapareciendo. Dieciséis días posterior de iniciado el estudio cambio de $40 \%$ a un $10 \%$. Sin embargo, el grupo de doma tradicional tardaron sesenta y dos días en disminuir la agresividad al igual que las acciones negativas. Probablemente este tipo de manejos agresivos generen una respuesta temerosa al manejo y como respuesta los animales pueden luchar, mostrar movimientos agitados, intentar escapar, vocalizar, mostrar tasas de respiración aumentadas, defecarse, mostrar cambios en las posiciones de sus oídos, cabeza y cola y expresiones faciales, además tratan de alejarse del área de manejo o del manejador (Haskell, Simm y Turner, 2014). Este tipo de manejo pudiera generar estrés crónico a los animales, lo cual tiene severas repercusiones en el comportamiento, por lo tanto, los animales mantienen la reactividad y siguen percibiendo al manejador como una amenaza.

76 | Pá g i n a
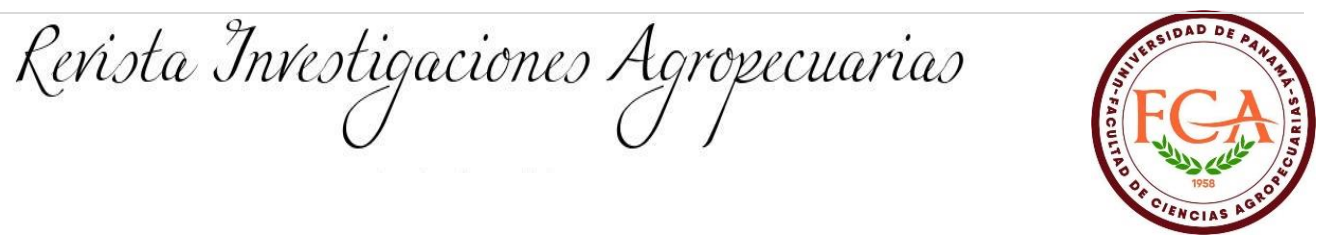


\section{Correlación entre variables de temperamento}

De manera general hubo correlación positiva entre score de brete y el score de salida $\left(\mathrm{r}_{\mathrm{s}}=0.43\right.$; $\mathrm{p}<0.001)$. En el caso de los animales del grupo de doma racional también hubo correlación estadísticamente significativa entre ambos scores $\left(\mathrm{r}_{\mathrm{s}}=0.44 ; \mathrm{p}<0.001\right)$. A su vez también hubo correlación entre ambos escores en el grupo de doma tradicional $\left(\mathrm{r}_{\mathrm{s}}=0.34 ; \mathrm{p}=0.001\right)$ (Tabla 1).

Tabla 1. Correlación entre variables de temperamento según método de doma empleado.

\begin{tabular}{ccc}
\hline $\begin{array}{c}\text { Variable } \\
\text { Temperamento }\end{array}$ & $\begin{array}{c}\text { Coeficiente de } \\
\text { correlación }\left(\mathbf{r}_{\mathbf{s}}\right)\end{array}$ & $\mathbf{p}$ \\
\hline Score de brete vs score de salida (General) & 0.43 & $<0.001$ \\
Score de brete vs score de salida (Racional) & 0.44 & $<0.001$ \\
Score de brete vs score de salida (Tradicional) & 0.34 & 0.001 \\
\hline
\end{tabular}

Los resultados indican que los scores utilizados para evaluar el temperamento (score de brete y salida) tuvieron correlaciones positivas y estadísticamente significativas en el grupo de doma racional y tradicional. Lo que sugiere que son indicadores prácticos para evaluar el temperamento en bovinos, lo cual coincide con lo reportado por Prayaga, (2003) donde también utilizó la velocidad de salida como una medida del temperamento. A pesar de que no evaluamos Glucocorticoides asociado con respuesta al estrés, existen reportes previos en donde la velocidad de salida del brete estuvo correlacionada con las concentraciones de cortisol en la sangre (Curley, 2004). Este estudio provee fuerte evidencia que el temperamento puede modificarse positivamente a través de acciones positivas mediante una adecuada relación humano-animal. Al mejorar este indicador se garantiza la seguridad de los animales y los manejadores del ganado. También es importante señalar que este rasgo es heredable (Burrow y Corbet, 2000; Haskell et al., 2014; Sant'Anna et al., 2013; Valente et al., 2017) y que existen correlaciones genéticas entre temperamento y rasgos de reproducción en vaquillas (Phocas et al., 2006). Además, nuestros resultados concuerdan con lo expuesto por Burrow (1997) al sugerir que la mejora del temperamento en los bovinos reduce el estrés experimentado durante los procedimientos de manejo.

A pesar de que son escasos los estudios donde se haya abordado el tema de la doma racional y sus implicaciones. Existe evidencia científica que la inadecuada interacción humano-animal puede generar estrés en los bovinos, experiencias de miedo, deshidratación y hambre; situación que produce fatiga y en ocasiones lesiones en los animales (Ferguson y Wagner, 2008). En ese sentido la doma racional tiene el potencial de reducir el estrés en los animales, convirtiéndose en una buena práctica de manejo tanto para los bovinos como para el humano. Estos resultados están en línea con lo expuesto por Destrez et al. (2018), donde reporta que la relación humano-animal es un 77 | Pá g i in a
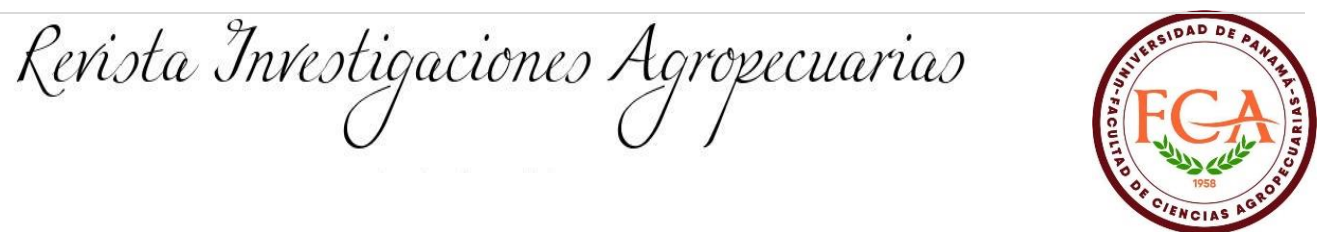
componente clave del bienestar humano y de los animales de granja. Por lo que interacciones negativas pueden tener repercusiones indeseables en el día a día en las fincas y en el producto final. En ese sentido nuestros resultados coinciden con una serie de investigaciones llevadas a cabo por (Breuer et al., 2000; Coleman, 2002), donde reportan la existencia de una relación directa entre la producción animal con las interacciones negativas. Además, estudios llevados a cabo por Hemsworth y Coleman (2011) indican que existe una relación secuencial entre las actitudes del ganadero, el comportamiento del ganadero, el comportamiento animal, la productividad y bienestar animal.

\section{Desempeño productivo de acuerdo con el método de doma empleado}

\section{Ganancia diaria de peso ( $\mathrm{kg} / \mathrm{d})$ en el tiempo de acuerdo con método de doma empleado}

La prueba de Kruskal-Wallis indica que no hubo diferencias estadísticamente significativas en la ganancia diaria de peso entre el grupo de doma racional y la tradicional $(\mathrm{K}-\mathrm{W}=12.94, \mathrm{p}=0.07)$. La ganancia diaria de peso para el grupo racional fue de $0.61 \pm 0.08 ; 0.61 \pm 0.08 ; 0.50 \pm 0.09$ y $0.45 \pm 0.04$ y en la tradicional fue de $0.06 \pm 0.19 ; 0.39 \pm 0.15 ; 0.33 \pm 0.11$ y $0.28 \pm 0.08$ durante los días dieciséis, treinta, sesenta y dos, ochenta y cinco respectivamente, Figura 2).

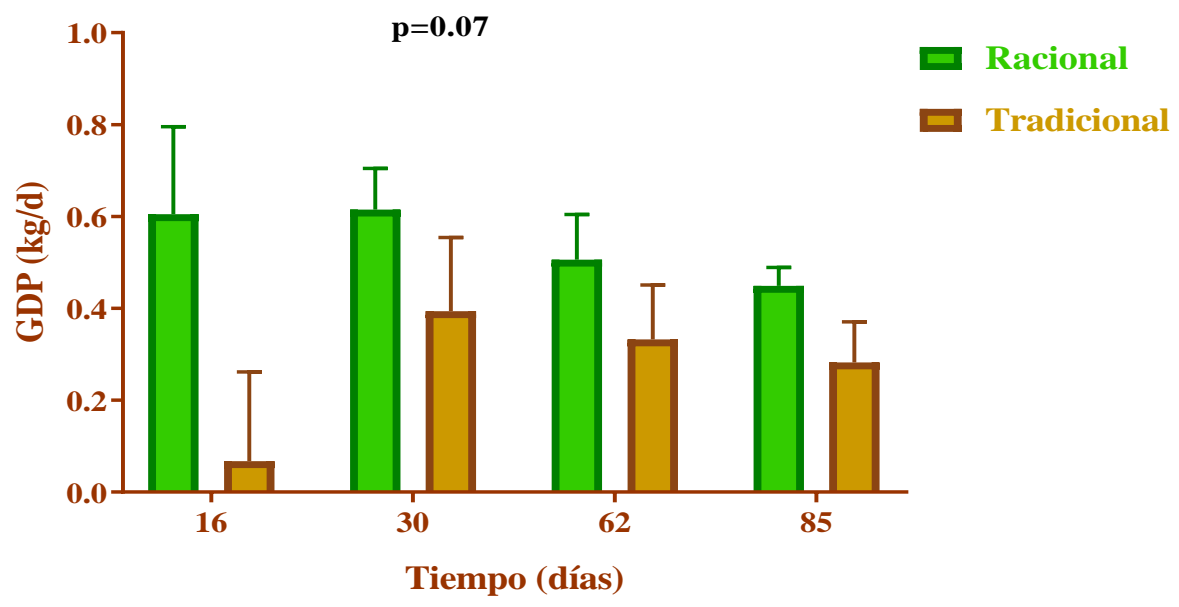

Figura 2. Media \pm error estándar de la ganancia diaria de peso en función del tiempo según el método de doma empleado. ${ }^{(p=0.07)}$ indica que no hubo diferencias estadísticamente significativas.

\section{Desempeño en función del peso final (kg) de acuerdo con método de doma empleado}

No se encontraron diferencias estadísticamente significativas (Mann-Whitney $U=31.50 ; p>0.05$ ) en el peso acumulado al final del periodo de estudio (85 días). Los animales a los cuales se les 


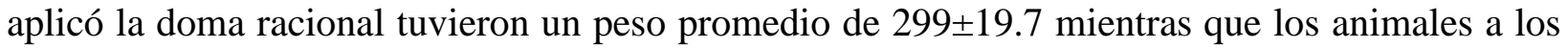
cuales se les aplico la doma tradicional tuvieron un peso promedio de 288.6 \pm 23.5 (Figura 3).

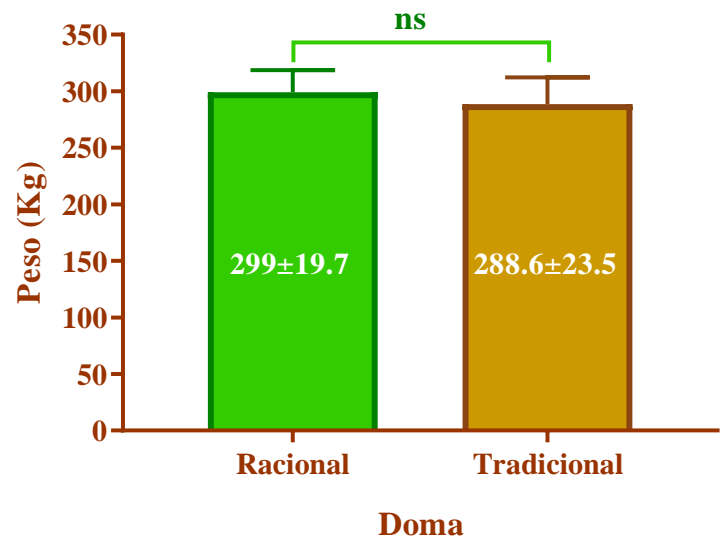

Figura 3. Media \pm error estándar del peso final a los 85 días según el método de doma empleado. ${ }^{(n s)}$ indica que no hubo diferencias estadísticamente significativas ( $p>0.05)$.

El desempeño productivo también se comprometió. Los valores medios al día dieciséis de la ganancia diaria de peso fueron significativamente superiores para los animales en la doma racional vs la tradicional, con un diferencial de $0,67 \mathrm{~g} / \mathrm{d}$. A pesar de que en las subsiguientes evaluaciones en el tiempo no fueron estadísticamente diferentes, siempre la ganancia diaria de peso fue numéricamente superior en el grupo que recibió la doma racional. Por su parte al evaluar el peso al final del estudio se obtuvo un margen de diferencia de $10.38 \pm 30.71 \mathrm{~kg}$ más de peso a favor de los animales que estaban en el grupo de doma racional vs la tradicional. Por lo tanto, estos resultados sugieren que al implementar la doma racional se puede reducir la reactividad o agresividad de los animales, manteniendo un óptimo desempeño productivo e implementando acciones positivas a través de una mejor relación humano-animal, ya que según Grandin (2014), uno de los principales tipos de problemas de bienestar animal está asociado con acciones humanas, debido a que los animales pueden reaccionar espontáneamente a las características humanas o pueden aprender a asociar la presencia y el comportamiento de los seres humanos con el tipo de manejo empleado (Waiblinguer et al., 2006).

Estudios llevados a cabo en otras latitudes coinciden con nuestros resultados, demostrado que el ganado con temperamento agresivo presenta menores ganancias diarias de peso (Burrow y Dillon 1997; Cafe et al., 2011; Müller y von Keyserlingk, 2006; Petherick et al., 2002, Sebastian et al., 2011), baja eficiencias de conversión alimenticia (Petherick et al.,2002), menor tiempo de alimentación y consumo de materia seca que aquellos con temperamento tranquilo (Cafe et al., 2011). Por su parte Voisinet et al. (1997) demostraron que el ganado de engorda con temperamentos agresivos producía carne de res dura y oscura. Lo que sugiere que el ganado con temperamento más tranquilo tiene una mejor calidad de la carne (Kadel et al., 2006; King et al., 79 | P á g i n a
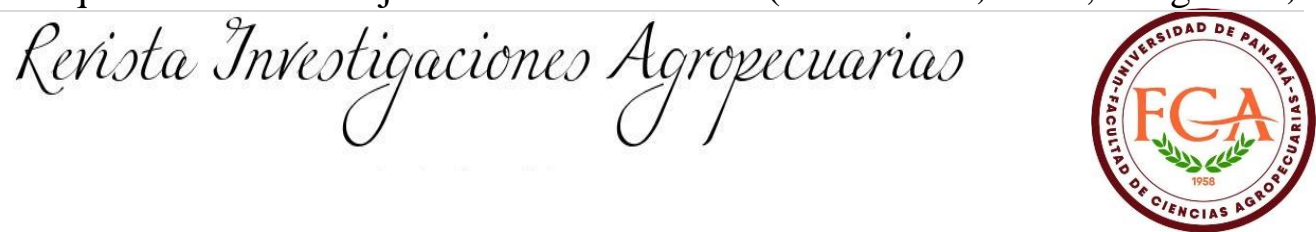
2006).

Tiempo por sesión y asistencias por semana dedicado a la doma de los animales por parte de los manejadores

El tiempo por sesión dedicado a la doma de los animales por parte de los manejadores no fue estadísticamente significativo entre grupos (Mann-Whitney $\mathrm{U}=35, \mathrm{p}>0.05$ ). Los animales del grupo doma racional y tradicional recibieron un manejo por parte de los manejadores de $1.49 \pm 0.26$ y 1.9 \pm 0.27 horas por sesión respectivamente (Figura 4A). Tampoco hubo diferencias estadísticamente significativas (Mann-Whitney $\mathrm{U}=16 ; \mathrm{p}>0.05$ ) en cuanto al número de veces que en que asistió la persona a darle manejo a los animales. El grupo de animales que recibieron la doma racional y tradicional tuvieron $3.4 \pm 0.17$ y $3.8 \pm 0.13$ número de asistencias/semana por los manejadores (Figura 4B).

(A)

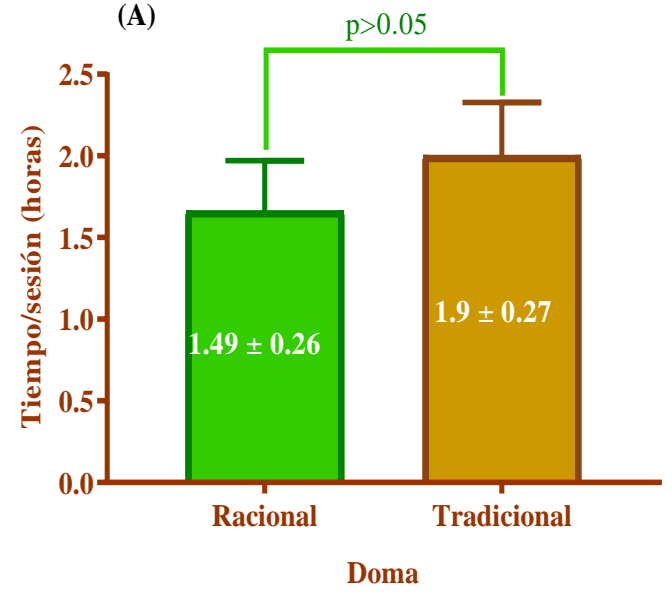

(B)

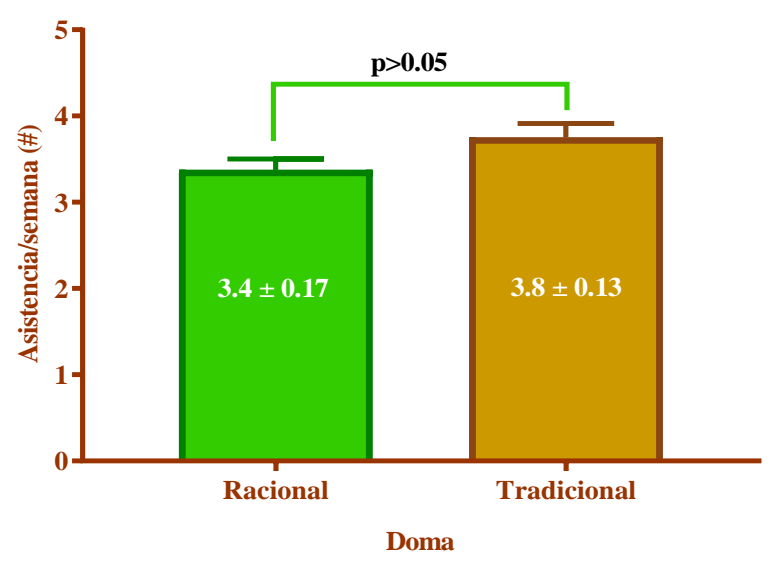

Figura 4. Tiempo por sesión (A) y asistencia por semana (B) dedicado a la doma de los animales por parte de los manejadores. ${ }^{(p>0.05)}$ indica que no hubo diferencias estadísticamente significativas $(p>0.05)$.

\section{Valoración económica según método de doma empleado}

Los resultados muestran que hubo diferencias estadísticamente significativas $\left(\chi^{2}=72.11 ; \mathrm{p}<0.001\right)$ en cuanto al dinero invertido al capital humano según el método de doma empleado. El método de doma racional y tradicional representó 850.08 y 1212.96 dólares respectivamente (Figura 5A). Estos resultados se obtuvieron en función del número de veces y las horas dedicas por semana al manejo de los animales durante el periodo de estudio. 
(A)

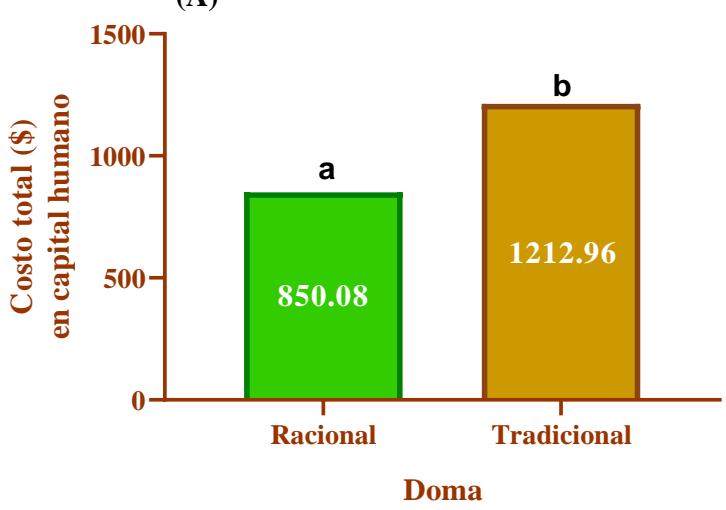

(B)

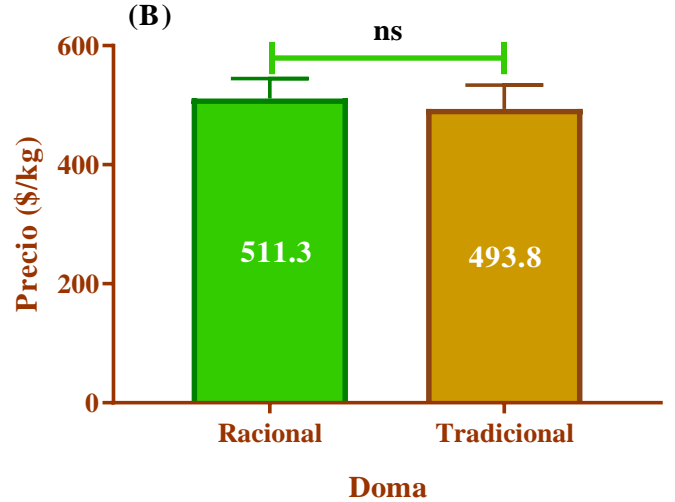

Figura 5. Media \pm error estándar del dinero invertido en cuanto al capital humano (A) y retorno económico de acuerdo con el peso final según método de doma empleado $(B)$. Letras diferentes ${ }^{(a, b)}$ indica diferencias altamente significativas $(p<0.01) y^{(n s)}$ indica que no hubo diferencias estadísticamente significativas $(p>0.05)$.

Los resultados muestran que no hubo diferencias estadísticamente significativas $(t=0.33 ; p=0.7)$ en cuanto al retorno económico según el peso de los animales de ambos grupos. Asumiendo un precio comercial establecido por la subasta ganadera de Panamá de 1.72 dólares/kg para la categoría de novillas en la época de diciembre de 2018. En este escenario los animales a los cuales se les aplicó la doma racional y tradicional generarían 511.3 y 493.8 dólares respectivamente (Figura 5B).

Además, se demostró que había diferencias estadísticamente significativas en la valoración económica del tiempo invertido en capital humano según método de doma, con un diferencial de 362.96 dólares a favor de la doma racional. Por su parte cuando se tradujo en términos económicos tomando en cuenta el peso al final de estudio de los animales, asumiendo un precio comercial; se determinó que los animales del grupo racional generarían en promedio 17.5 dólares más por animal que los del grupo de doma tradicional. Es decir que además de los beneficios antes expuestos este tipo de manejo racional tiene implicaciones económicas positivas para los ganaderos.

En conclusión, la doma racional tuvo un efecto positivo sobre el temperamento, interacción humano-animal y en el desempeño productivo. Además, de tener implicaciones positivas en términos económicos. Con lo cual queda demostrado que es fundamental capacitar al personal en buenas prácticas de manejo del ganado para ofrecer a los animales una vida que valga la pena ser vivida, lo cual se puede traducir en un solo bienestar (Humano- animal y ambiente). 


\section{AGRADECIMIENTOS}

Agradecemos al profesor y estudiantes del Curso de Juzgamiento de Ganado Bovino (LCPA 430), Departamento de Zootecnia de la Universidad de Panamá por su colaboración en este estudio.

\section{REFERENCIAS BIBLIOGRÁFICAS}

Burrow, H. M. (1997). Measurements of temperature and their rela-tionships with performance traits of beef cattle. Animal Breeding Abstracts, 64, 477-495.

Burrow, H. M. y Corbet, N. J. (2000). Genetic and environmental factors affecting temperament of zebu and zebu-derived beef cattle grazed at pasture in the tropics. Australian Journal of Agricultural Research, 55, 155-162.

Burrow, H. M. y Dillon, R. D. (1997). Relationships between temperament and growth in a feedlot and commercial carcass. traits of Bos indicus crossbreds. Australian Journal of Agricultural Research, 37, 407-411.

Breuer, K., Hemsworth, P. H., Barnett, J. L., Matthews, L. R. y Coleman, G. J. (2000). Behavioural response to humans and the productivity of commercial dairy cows. Applied Animal Behaviour Science 66, 273-288.

Cafe, L. M., Robinson, D. L., Ferguson, D. M., McIntyre, B. L., Geesink, G. H. y Greenwood, P. L. (2011). Cattle temperament: persistence of assessments and associations with productivity, efficiency, carcass and meat quality traits. Journal of Animal Science, 89, 14521465.

Coleman, G. J. y Hemsworth, P. H. (2014). Training to improve stockperson beliefs and behaviour towards livestock enhances welfare and productivity. Revue Scientifique et Technique33, 131-137.

Cziszter, L. T., Gavojdian, D., Neamt, R., Neciu, F., Kusza, S., y Ilie, D. E. (2016). Effects of temperament on production and reproductive performances in Simmental dual-purpose cows. Journal of Veterinary Behavior: Clinical Applications and Research, 15, 50-55.

Cooke, R. F., Schubach, K. M., Marques, R. S., Peres, R. F. G., Silva, L. G. T., Carvalho, R. S., ... Vasconcelos, J. L. M. (2017). Effects of temperament on physiological, productive, and reproductive responses in beef cows. Journal of Animal Science, 95(1), 1.

Cooke, R. F., Bohnert, D. W., Cappellozza, B. I., Mueller, C. J., y del Curto, T. (2012). Effects of temperament and acclimation to handling on reproductive performance of Bos taurus beef females. Journal of Animal Science, 90(10), 3547-3555.

Cooke, R., Bohnert, D., Meneghetti, M., Losi, T. y Vasconcelos, J. (2011). Effects of temperament on pregnancy rates to fixed-timed AI in Bos indicus beef cows. Livestock Science, 142(1-3), 108-113.

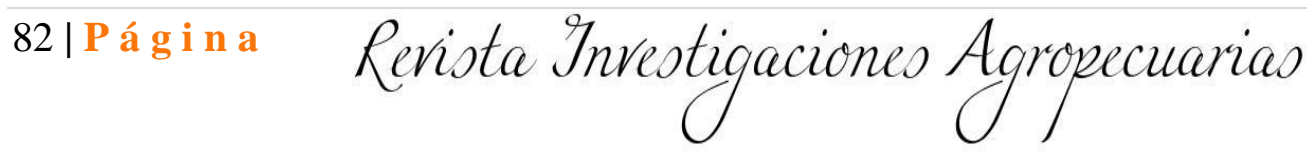


Ceballos, M. C., Sant'Anna, A. C., Góis, K. C. R., Ferraudo, A. S., Negrao, J. A., y da Costa, M. J. R. P. (2018). Investigating the relationship between human-animal interactions, reactivity, stress response and reproductive performance in Nellore heifers. Livestock Science, 217, 6575.

Curley, K. O. Jr. (2004). Influence of temperament on bovine hypothalamic-pituitary-adrenal function. Master's thesis, Texas A\&M University. Texas A\&M University. Available electronically from http: //hdl .handle .net /1969.1/3212.

Destrez, A., Haslin, E. y Boivin, X. (2018). What stockperson behavior during weighing reveals about the relationship between humans and suckling beef cattle: A preliminary study. Applied Animal Behaviour Science, 209, 8-13.

Ferguson, D. M. y Warner, R. D. (2008). Have we underestimated the impact of pre-slaughter stress on meat quality in ruminants? Meat Science, 80, 12-19.

García-Pinillos, R., Appleby, M. C., Scottpark, F. y Smith, C. W. (2015) One Welfare. Veterinary Record 177, 629-630.

Grandin T. (2014). Animal welfare and society concerns finding the missing link. Meat Science 98(3), 461-9.

Grandin, T. (1993a). Behavioral agitation during handling of cattle is persistent over time. Applied Animal Behaviour Science, 36,1-9.

Grandin T, (1993b). Handling Facilities and Restraint of Range Cattle. In: Grandin T (ed.), Livestock Handling and Transport. CAB International, Oxon, Wallingford, UK, pp. 75-94.

Haile-Mariam, M., Bowman, P. J. y Goddard, M. E. (2004). Genetic parameters of fertility traits and their correlation with production, type, workability, live weight, survival index, and cell count. Australian Journal of Agricultural Research, 55, 77-87.

Haskell, M. J., Simm, G. y Turner, S. P. (2014). Genetic selection for temperament traits in dairy and beef cattle. Frontiers in Genetics, 5, 1-18.

Hedlund, L. y Løvlie, H. (2015). Personality and production: Nervous cows produce less milk. Journal of Dairy Science, 98(9), 5819-5828.

Hemsworth, P. H. y Coleman, G. J. (2011). Human-livestock interactions: The stockperson and the productivity of intensively farmed animals. 2nd Ed. CABI Publishing, Wallingford, UK.

Hemsworth, P. H. (2007). Ethical stockmanship. Australian veterinary Journal, 85, 194-200.

Kadel, M. J., Johnston, D. J., Burrow, H. M., Graser, H. U. y Ferguson, D. M. (2006). Genetics of flight time and other measures of temperament and their value as selection criteria for improving meat quality traits in tropically adapted breeds of beef cattle. Australian Journal of Agricultural Research, 57, 1029-1035.

King, D. A., Schuehle Pfeiffer, C. E., Randel, R. D., Welsh, Jr. T. H.,Oliphint, R. A., Baird, B. E., Curley, Jr., K. O., Vann, R. C., Hale, D. S. y Savell, J. W. (2006). Influence of animal temperament and stress responsiveness on the carcass quality and beef tenderness of feedlot cattle. Journal Meat Science, 74, 546-556.

83|Pág in a Revista Investigaciones Agropecuarias 
Koolhaas, J. M., Korte, S. M., De Boer, S. F., van der Vegt, B. J., van Reenen, C. G., Hopster, H., De Jong, I. C., Ruis, M. A. W. y Blockhuis, H. J. (1999). Coping styles in animals: Current status in behavior and stress-physiology. Neuroscience \& Biobehavioral Reviews, 23, 925935.

Oyeka C. A. (2010). An Introduction to Applied Statistical Methods. (8th edn), Nobern Avocation Publishing Company, Enugu, Nigeria.

Petherick, J. C., Holroyd, R. G., Doogan, V. J. y Venus B. K. (2002). Productivity, carcass and meat quality of lot-fed Bos indicus cross steers grouped according to temperament. Australian Journal of Agricultural Research, 42, 389-398.

Phocas, F., Boivin, X., Sapa, J., Trillat, G., Boissy, A. y Le Neindre, P. (2006). Genetic correlations between temperament and breeding traits in Limousin heifers. Animal Science, 82, 805-811.

Reale, D., Gallant, B. Y., Leblanc, M. y Festa-Bianchet, M. (2000). Consistency of temperament in bighorn ewes and correlates with behavior and life history. Animal Behaviour, 60, 589597.

Reinhardt, C. D., Busby, W. D. y Corah, L. R. (2009). Relationship of various incoming cattle traits with feed lot performance and carcass traits. Journal of Animal Science, 87, 3030-3042.

Santos, F., Jayme, D., Dornellas de Oliveira, N., Jayme, C. y Pereira T. (2015). O comportamento do homem perante os bovinos determina sua resposta frente à produtividade, podendo esta ser positiva ou negativa de acordo com o tratamento dado aos animais. Doma Racional de bovinos. Caderno Técnicos de Veterinária e Zootecnia, 9-18.

Sebastian, T., Watts, J., Stookey, J. M Buchanan, F. y Waldner, Ch. (2011). Temperament in beef cattle: Methods of measurement and their relationship to production. Canadian Journal of Animal Science, 91(4), 557-565.

Sewalem, A., Miglior, F. y Kisteman, G. J. (2011). Genetic parameters of milking temperament and milking speed in Canadian Holsteins. Journal of Dairy Science, 94, 512-516.

Sewalem, A., Miglior, F. y Kistemaker, G. J. (2010). Analysis of the relationship between workability traits and functional longevity in Canadian dairy breeds. Journal of Dairy Science, 93, 4359-4365.

Sutherland, M. A. y Dowling, S. K. (2014). The relationship between responsive- ness of firstlactation heifers to humans and the behavioural response to milkingand milk production measures. Journal of Veterinary Behavior: Clinical Applications and Research, 9, 30-33.

Turner, S. P., Navajas, E. A., Hyslop, J. J., Ross, D. W., Richardson, R. I. y Prieto, N. (2011). Associations between response to handling and growth and meat quality in frequently handled Bos Taurus beef cattle. Journal of Animal Science, 89, 4239-4248

Valente, T. S., Albito, O. D., Sant'Anna, A. C., Carvalheiro, R., Baldi, F., Albuquerque, L. G., y da Costa, M. J. R. P. (2017). Genetic parameter estimates for temperament, heifer rebreeding, and stayability in Nellore cattle. Livestock Science, 206, 45-50.

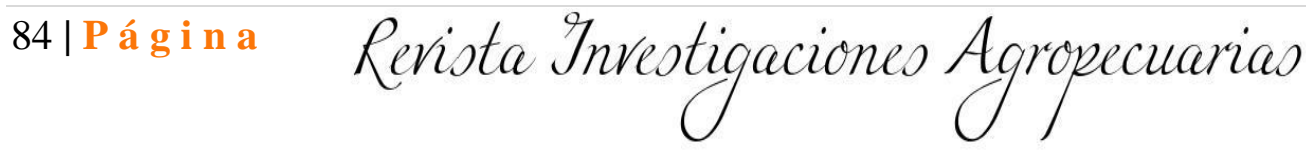


Voisinet, B. D., Grandin, T., Tatum, J. D., O'Connor, S. F. y Struthers, J. J. (1997). Feedlot cattle with calm temperaments have higher average daily gains than cattle with excitable temperaments. Journal of Animal Science, 75, 892-896. 